\title{
Actividad prefrontal y alcoholismo de fin de semana en jóvenes
}

\author{
Luis Miguel García-Moreno*; Javier Expósito*; Claudia Sanhueza*; Maa Teresa Angulo** \\ * Sección Departamental de Psicobiología. Facultad de Educación. Universidad Complutense de Madrid. \\ ** Escuela Universitaria de Enfermería, Fisioterapia y Podología. Facultad de Medicina. Universidad Complutense de Madrid. \\ Enviar correspondencia a: \\ Luis Miguel García Moreno. Sección Departamental de Psicobiología. Facultad de Educación. Universidad Complutense de Madrid. \\ C/ Rector Royo Villanova, s/n. 28040 Madrid. E-mail: luismgm@edu.ucm.es.
}

Recibido: Agosto de 2007

Aceptado: Mayo de 2008

\section{RESUMEN}

Antecedentes. El consumo elevado de alcohol por parte de jóvenes durante el fin de semana se está convirtiendo en un problema social y familiar importante pero también en un problema de salud considerable. El consumo abusivo ocasiona daños en el cerebro pero con diferente distribución, ya que la corteza prefrontal (CP) es una de las regiones que resulta más afectada.

Objetivos. Hay numerosos estudios sobre los efectos del consumo crónico de alcohol. Nuestro objetivo es determinar los déficits neuropsicológicos en la actividad prefrontal ocasionados por el consumo abusivo de alcohol de manera intermitente en adolescentes.

Método. Se realizó una evaluación neuropsicológica a los sujetos para valorar su rendimiento en diversas tareas que implican a la CP. Participaron 62 sujetos (edad media 18.82 \pm 1.099 ) distribuidos en tres grupos: 1) Jóvenes que consumían alcohol de manera abusiva durante los fines de semana (ALE); 2) Jóvenes que consumían más moderadamente durante los fines de semana (ALM); y 3) Jóvenes que no consumían alcohol (CTR).

Resultados y Conclusiones. Los resultados indican que el consumo abusivo intermitente de alcohol por parte de jóvenes, tanto de manera elevada como moderada, provoca un peor rendimiento en tareas neuropsicológicas como Dígitos, Corsi o Stroop, las cuales dependen de un correcto funcionamiento de la CP. Nuestros resultados apoyan la idea de que este patrón de consumo provoca un deterioro neurocognitivo y neuroconductual similar en muchos aspectos al observado en bebedores crónicos. Además, el consumo abusivo de fin de semana por parte de adolescentes y jóvenes incrementa el riesgo de desarrollar dependencia alcohólica y otras patologías en etapas posteriores de la vida.

Palabras clave: Adolescencia, alcohol, consumo crónico intermitente, corteza prefrontal, funciones ejecutivas.

\section{ABSTRACT}

Background. High alcohol consumption by young people at weekends is a public health problem of considerable social and family importance. Chronic alcohol intake causes structural changes in the central nervous system, though the effects are not uniform throughout the brain. The prefrontal cortex (PC) has been reported to be one of the regions most sensitive to prolonged alcohol ingestion.

Objectives. The effects of chronic ethanol exposure have been extensively studied. Our aim is to identify the neuropsychological deficits in PC function induced by intermittent heavy alcohol drinking in young adults.

Methods. Neuropsychological assessment was carried out on participants to examine their performance in PC-dependent tasks. 62 subjects (mean age 18.82 1 1.099) were assigned to one of three categories: 1) Those reporting heavy binge drinking of alcohol at weekends (ALE); 2) Those reporting moderate binge drinking of alcohol at weekends (ALM); and 3) Those reporting no alcohol intake (CTR).

Results and Conclusions. The findings indicate that intermittent alcohol binge drinking, either heavy or moderate, in young people results in poorer performance in neuropsychological tasks such as Digits, Corsi or Stroop, which depend on correct PC functioning. Our results support the claim that the binge pattern of exposure to ethanol leads to neurocognitive and neurobehavioural impairment equivalent, in many respects, to that found in chronic drinkers. Moreover, intermittent heavy alcohol drinking in adolescence and early adulthood increases the risks of lifetime alcohol dependence and other psychopathologies.

Key words: Adolescence, alcohol, chronic intermittent intake, executive functions, prefrontal cortex 


\section{INTRODUCCIÓN}

A ctualmente, los jóvenes se inician en el consumo de bebidas alcohólicas cada vez a una edad más temprana para, posteriormente, alcanzar niveles de consumo superiores con el consiguiente riesgo de desarrollar dependencia alcohólica. Se estima que un $40 \%$ de adolescentes que comienzan a beber antes de los 15 años de edad, desarrollarán problemas relacionados con el consumo de alcohol en edades posteriores (Zeigler, Wang, Yoast, Dickinson, McCaffree, Robinowitz et al., 2005). En España el alcohol provoca la adicción con mayor prevalencia, el $87 \%$ de la población ha consumido esta sustancia en alguna ocasión, entre los 15 y los 65 años, el 47\% consume con una frecuencia semanal y un $13 \%$ diariamente. En la actualidad son muy frecuentes las borracheras de fin de semana (episodios breves, horas o días, de alto consumo de alcohol), sobre todo por la famosa "moda del botellón" que reúne a jóvenes en espacios públicos de la ciudad en torno al alcohol (Cadaveira y Corral, 2005; Garrido y Fernández-Guinea, 2004; Sánchez, 2002) y donde su consumo es la principal motivación que reúne a los jóvenes (Calafat, Juan, Becoña, Castillo, Fernández, Franco et al., 2005). Todo ello viene agravado por la peculiar relación que los jóvenes establecen con el consumo de alcohol ya que, si bien algunos son conscientes de los posibles efectos negativos que ocasiona el consumo, una gran mayoría le atribuye efectos positivos que favorecen la socialización y la desinhibición (Pascual, 2002). Las consecuencias negativas que puede provocar el consumo abusivo son de índole muy diversa y pueden ir desde la dependencia alcohólica hasta llegar, incluso, a la muerte (Zeigler et al., 2005).

La adolescencia es un periodo crítico de desarrollo que constituye la transición de la infancia a la edad adulta y que, de manera general, se situaría entre los 10 y los 25 años de edad. A nivel cerebral, es una etapa en la que las áreas corticales frontales experimentan los mayores cambios en cuanto a receptores sinápticos, densidad y mielinización axonal se refiere. Estos procesos de remodelación y desarrollo de la plasticidad cerebral se producen con el objeto de adaptar los circuitos neuronales y sus conexiones sinápticas a las necesidades del ambiente en el que se desarrolla el adolescente. El cortex prefrontal y el sistema límbico, que incluye estructuras como el hipocampo, la amígdala, el núcleo acccumbens y el hipotálamo entre otras, son sometidos a esta reorganización durante la adolescencia. Es por tanto un periodo evolutivo, que dadas sus características, hace más vulnerable al cerebro a las agresiones externas (Carpenter-Hyland y Chandler, 2007; Crews, He y Hodge, 2007).

El alcohol puede alterar tanto la estructura como la función del Sistema Nervioso Central (SNC) de manera significativa provocando la aparición de un cierto grado de deterioro neuropsicológico (Rosenbloom, Sullivan y Pfefferbaum, 2003). Sin embargo, este deterioro puede presentar una alta variabilidad debido a causas tales como la cantidad de alcohol consumida, los antecedentes y el patrón de consumo, el consumo concomitante de otras sustancias tóxicas y adictivas o las características intrínsecas del consumidor (estado premórbido, enfermedades sistémicas, el estado nutricional, etc.) (Corral-Varela y Cadaveira, 2002; Rosenbloom et al., 2003; Zeigler et al., 2005). La existencia de factores de riesgo tales como lesiones cerebrales previas, cuadros epilépticos o el consumo de otras drogas de manera simultánea al consumo de alcohol pueden estar en la base de muchas de las alteraciones cerebrales ocasionadas por el consumo abusivo, sin embargo, para algunos autores, la abstinencia alcohólica es la responsable más directa de la mayoría de dichas alteraciones (Lukoyanov, Madeira y Paula-Barbosa, 1996; Neiman, 1998; Rathlev, Ulrich, Delanty y D'Onofrio, 2006). Con respecto al patrón de consumo, estudios recientes sobre el consumo intermitente de alcohol en ratas seudoadolescentes, han demostrado la existencia de un importante daño inflamatorio cerebral refrendado por los hallazgos de un aumento de las muertes celulares en zonas del neocortex, hipocampo y cerebelo, que originarían alteraciones del comportamiento cerebral a largo plazo y que afectarían a los procesos cognitivos y motores (Pascual, Blanco, Cauli, Minarro y Guerri, 2007).

El consumo crónico de alcohol puede ocasionar deterioro neurológico y cognitivo en humanos. Dicho consumo produce alteraciones morfológicas y neuroquímicas en el sistema nervioso central tanto de humanos (Charness, 1993) como en animales de experimentación (Walker, Hunter y Abraham, 1981) y ocasiona modificaciones estructurales en el SNC aunque estos efectos no son uniformes en las diferentes estructuras cerebrales. Los cambios que ocurren en regiones como el hipocampo o el neocortex son de especial relevancia porque son estructuras implicadas en procesos como la atención, memoria o procesos ejecutivos, los cuales están alterados en el alcoholismo (Beracochea, Durkin y Jaffard, 1986; Victor, 1994), de hecho, en torno a un $75 \%$ de alcohólicos manifiestan disfunciones neuropsicológicas significativas (Landa, Fernández-Montalvo y Tirapu, 2004; Roehrich y Goldman, 1993) aunque algunos resultados con neuroimagen por Resonancia Magnética se ha demostrado que la mayor reducción de volumen cerebral como consecuencia del consumo de alcohol ocurre en los lóbulos frontales y en otras estructuras como el hipocampo, el tálamo o el cerebelo (Moselhy, Georgiou y Kahn, 2001; Pfefferbaum, Sullivan, Mathalon y Lim, 1997; Sullivan, 2000), estructuras que están estrechamente relacionadas con procesos como la memoria y el aprendizaje, la atención, la resolución de problemas o la organización de la conducta. 
En la actualidad ha surgido un especial interés por el estudio de las funciones ejecutivas, consideradas como los procesos mentales que ponemos en marcha para resolver problemas generados interna y externamente, es decir, las representaciones mentales del sujeto y los problemas derivados de su interacción con el medio (Crews et al., 2007). El objetivo de las funciones ejecutivas es solucionar estos problemas de una forma eficaz y aceptable para la persona y la sociedad (Papazian, Alfonso y Luzondo, 2006). Están implicadas en tareas como la anticipación y el establecimiento de metas, el diseño de planes, la inhibición de respuestas inapropiadas, la adecuada selección de conductas y su organización en el espacio y en el tiempo, la flexibilidad cognitiva en la monitorización de estrategias, la supervisión de las conductas en función de estados motivacionales y afectivos y la toma de decisiones (Damasio, 1996; Stuss y Alexander, 2000). Todas estas funciones se asocian neuroanatómicamente a circuitos neurales entre los que los de la corteza prefrontal juegan un papel fundamental. Se han descrito al menos dos circuitos funcionales dentro del Cortex prefrontal que poseen importantes connotaciones neuropsicológicas (Bechara, Damasio y Damasio, 2000; Cummings, 1993; Damasio, 1996), por una parte, el circuito dorsolateral implicado en habilidades cognitivas, como la memoria de trabajo, la atención selectiva, la formación de conceptos o la flexibilidad cognitiva. Por otra parte, estaría el circuito ventromedial que se asocia al procesamiento de señales somático-emocionales que actúan como indicadores motivacionales en los procesos de toma de decisiones hacia objetivos socialmente adaptativos (Bechara, Damasio y Damasio, 2000; Damasio, 1996; Verdejo, Orozco, Meersmans, Aguilar y Pérez-García, 2004). Los efectos del consumo crónico de alcohol sobre la corteza prefrontal y las funciones ejecutivas han sido estudiados por numerosos investigadores, sin embargo, el consumo abusivo agudo intermitente lo ha sido menos. En nuestro trabajo queremos comprobar los efectos del consumo de alcohol durante el fin de semana, que sigue un patrón de consumo agudo intermitente, sobre determinadas capacidades neuropsicológicas vinculadas a la actividad neural de la corteza prefrontal.

\section{MÉTODOS}

\section{Sujetos}

Participaron en el estudio 62 estudiantes universitarios menores de 20 años y alumnos de primer curso de carrera $(52$ mujeres y 10 hombres, media de edad 18.82 1 1.099) repartidos en tres grupos según su patrón de consumo de alcohol durante el fin de semana: ALE $(n=20)$, consumo elevado de alcohol,
ALM $(n=20)$, consumo moderado de alcohol y CTR $(n=22)$, alumnos que no consumían nada de alcohol. Por supuesto, y dada las características del estudio, ninguno de los sujetos seleccionados consumía alcohol entre semana, por este motivo, a lo largo del presente artículo, siempre que nos refiramos al consumo de alcohol entre los sujetos de nuestra muestra, se entenderá que es en fines de semana únicamente. Para determinar este patrón, los alumnos contestaron a un cuestionario sobre salud, actitudes y ocio juvenil (ESAJ, Encuesta Sobre Actitudes en la Juventud) que recopila de forma retrospectiva información de diferentes aspectos biológicos, psicológicos y emocionales de cada individuo: antecedentes familiares y médicos, hitos del desarrollo, historia educativa, hábitos de vida y de ocio, actitudes, etc. Uno de los apartados del ESAJ recaba información sobre el consumo de alcohol, su frecuencia e intensidad e incluye diferentes cuestiones para garantizar la fiabilidad de estas respuestas. Los sujetos seleccionados presentaban un perfil homogéneo en aquellos aspectos no referidos al consumo de alcohol.

\section{Evaluación neuropsicológica}

Para evaluar funciones cognitivas ligadas a la actividad de la corteza prefrontal del cerebro hemos seleccionado una serie de pruebas, algunas específicas y otras incluidas a su vez en pruebas estandarizadas y diseñadas para evaluar cualidades mentales más amplias. Todos los sujetos fueron evaluados en sesiones individuales de unos 60-70 minutos de duración. Las pruebas consideradas fueron las siguientes:

TAVEC: El Test de aprendizaje verbal España-Complutense evalúa aprendizaje verbal en tareas de recuerdo inmediato y diferido y reconocimiento. Entre los diferentes parámetros que se evalúan en esta prueba se encuentran el número de palabras repetidas que el sujeto incluye en su recuerdo de las listas de palabras, las intrusiones, es decir, palabras que el sujeto incluye pero que no figuran en la lista a recordar y los falsos positivos, palabras reconocidas como pertenecientes a una lista y que no lo son.

Torre de Hanoi: Consta de un tablero con tres pivotes verticales ( $A, B$ y $C$ ), en uno de los cuales se apilan cinco aros de tamaño decreciente formando una pirámide. El objetivo de la tarea es desplazar todos los discos, moviéndolos de uno en uno cada vez, de la posición $\mathrm{A}$ a la $\mathrm{C}$ y de manera que formen la misma pirámide y sin que en ninguna de las posiciones intermedias un disco mayor esté colocado sobre uno más pequeño (Tirapu-Ustárroz, Muñoz-Céspedes, PelegrínValero y Albéniz-Ferreras, 2005). Se evaluó el tiempo empleado y el número de movimientos empleados en la resolución. 
Dígitos de la escala de memoria de Wechsler y Cubos de Corsi: Estas prueban permiten evaluar el recuerdo inmediato con material verbal (Dígitos) o visoespacial (Cubos de Corsi) en sentido directo o inverso. Se evaluó el número de series de elementos de dificultad creciente resuelto de manera correcta.

Test Stroop de colores y palabras: Diseñado para evaluar la capacidad para evitar respuestas generadas automáticamente gracias a un proceso de inhibición. Consta de palabras que denominan colores, colores puros y palabras coloreadas. El sujeto debe nombrar el color de la tinta de una palabra que denomina un color distinto. El objetivo de la tarea es medir la capacidad de una persona para generar un control inhibitorio sobre estímulos predominantes y automatizados, para permitir la emisión de respuestas programadas a través del esfuerzo cognitivo. Se evaluó el número de elementos resuelto correctamente (leer palabra o denominar color) en cada ensayo.

Recitado de series (Serie numérica): Los sujetos debían, a partir de un número de tres dígitos dado por el evaluador, sumar 7 y al resultado restarle 3 verbalizando el resultado de cada operación durante 30 segundos. Se valoró el número y porcentaje de operaciones correctas realizadas.

Los resultados obtenidas por los sujetos en las diferentes pruebas fueron analizadas con el programa estadístico SigmaStat $@$ 3.0.1. (1992-2003 SPSS Inc) y para ello se utilizo un ANOVA de un factor $y$, para comprobar que grupos diferían entre sí, el método de Student-Newman-Kuls.

\section{RESULTADOS}

En el TAVEC hemos observado que los jóvenes que consumen alcohol mostraron una precisión mnésica significativamente peor que los jóvenes control ya que cometieron muchas más perseveraciones (repetir palabras ya dichas) (PRL: $F_{2,61}=10.027, p<0.001$ ) y muchas más intrusiones (falsos recuerdos) (IRL: $F_{2,61}=4.543, p<0.05$ ) en las pruebas de recuerdo libre y también en la de reconocimiento, ya que cometieron más falsos positivos que los jóvenes del grupo control (FP: $F_{2,61}=6.210, p<0.001$ ) (Figura 1). En el recuerdo inmediato de palabras, los sujetos del grupo control recordaron más palabras, en el primer ensayo, que los jóvenes que consumían alcohol (RIA1: $F_{2,61}=6.742$, $\mathrm{p}<0.01)$. Sin embargo, el efecto de la repetición tuvo un efecto positivo sobre el aprendizaje de palabras tanto en los jóvenes que consumían alcohol como en los abstemios ya que en el quinto ensayo para recordar la misma lista de palabras, los tres grupos mostraron un rendimiento similar.

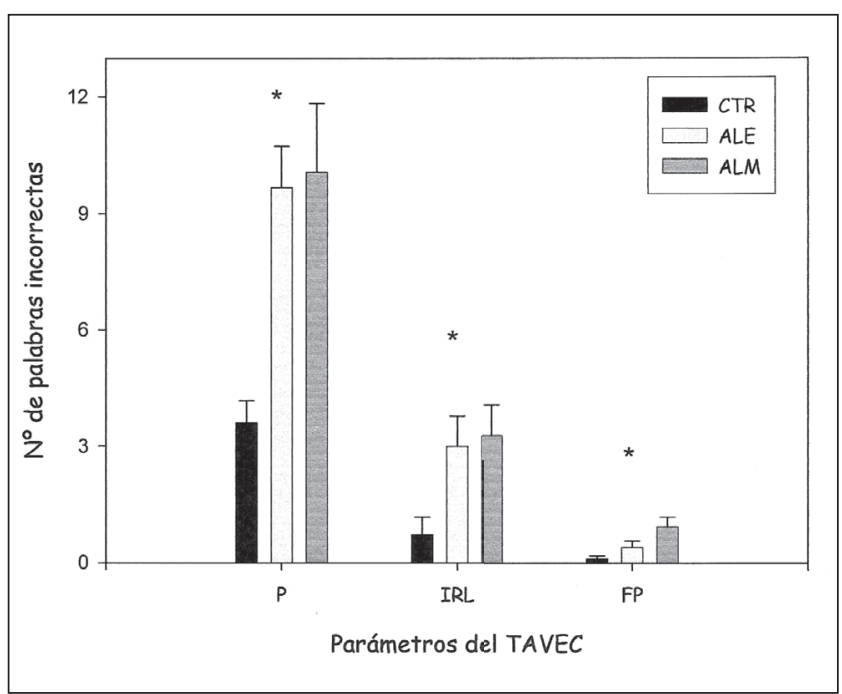

Figura 1. Precisión en memoria de palabras. Los sujetos del grupo control (CTR) come-ten menos imprecisiones en recuerdo y reconocimiento de palabras que los sujetos que consumen alcohol (ALE y ALM). (Abreviaturas: $P=$ perseveraciones en la prueba de recuerdo libre, IRL= intrusiones en recuerdo libre, $\mathrm{FP}=$ falsos positivos en reconoci-miento). (* Significatividad mínima $p<0$ '05).

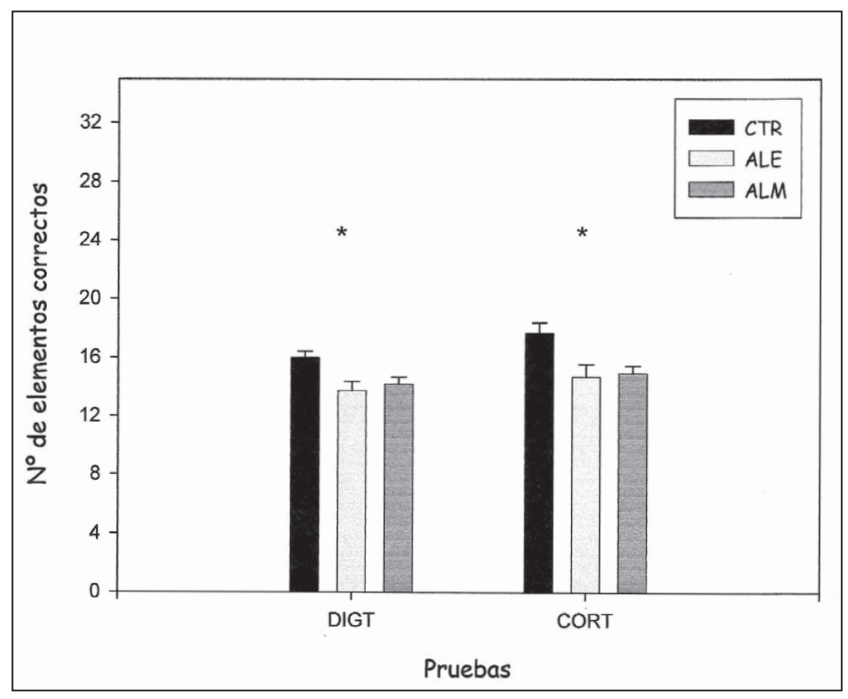

Figura 2. Resultados en las pruebas de Dígitos (DIGT) y Cubos de Corsi (CORT). Se observa un mejor rendimiento de los sujetos que no consumen alcohol. (* Significativi-dad mínima p<0’05).

En las pruebas de memoria inmediata o amplitud atencional también se observa una tendencia parecida. Así en la prueba de dígitos, los jóvenes del grupo CTR obtuvieron mejores puntuaciones totales que los grupos ALE y ALM (DT: $F_{2,61}=5.783, p<0.01$ ) aunque en la prueba de recuerdo de dígitos en orden inver- 
so no hubo diferencias significativas entre los grupos a pesar de que el grupo CTR fue superior a los que consumieron alcohol. Algo similar ocurrió en la prueba de Corsi, donde los jóvenes del grupo control obtuvieron mejores puntuaciones que los grupos que consumían alcohol (CRST: $\left.F_{2,61}=5.681, p<0.01\right) y$, donde, una vez más no hubo diferencias significativas en el recuerdo en orden inverso (Figura 2). En cuanto a la capacidad de inhibición de respuestas automatizadas que evaluamos con el test de Stroop, los jóvenes abstemios obtuvieron mejores rtesultados tanto en la lectura de palabras (SLP: $F_{2,61}=6$ '966, $p<0$ 001) como en la denominación de colores (SDC: $F_{2,61}=11.841$, $\mathrm{p}<0.0001)$. También el grupo CTR fue más resistente a la interferencia que los grupos ALE y ALM ya que fueron capaces de denominar un número mayor de colores cuando no había concordancia con la palabra escrita (SPC: $F_{2,61}=6.954, p<0.01$ ) (Figura 3). En la serie numérica el grupo de jóvenes no consumidores obtuvo un rendimiento significativamente mayor que los grupos de jóvenes consumidores ya que, no solo realizaron un mayor número de operaciones en total (SOT: $F_{2,61}=4.537, p<0.05$ ), sino que también obtuvieron un porcentaje superior de operaciones realizadas correctamente (SPOC: $F_{2,61}=3.961, p<0.05$ ) (Figura 4). Por último, en el Test de la Torre de Hanoi no se encontraron diferencias significativas a pesar de que los alumnos del grupo control obtuvieron mejores resultados que aquellos que consumían alcohol en el fin de semana.

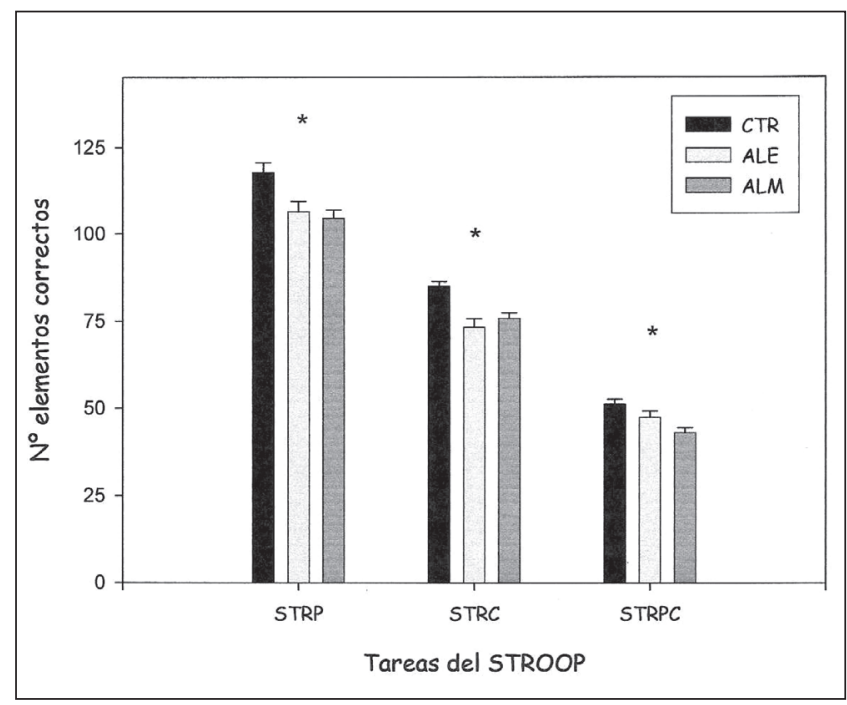

Figura3. Rendimiento en el test de Stroop. Los jóvenes bebedores obtienen peores re-sultados en las tres pruebas. (Abreviaturas: STRP= lectura de palabras, STRC= denomi-nación de colores, STRPC= palabra-color) (* Significatividad mínima $p<0$ '05).

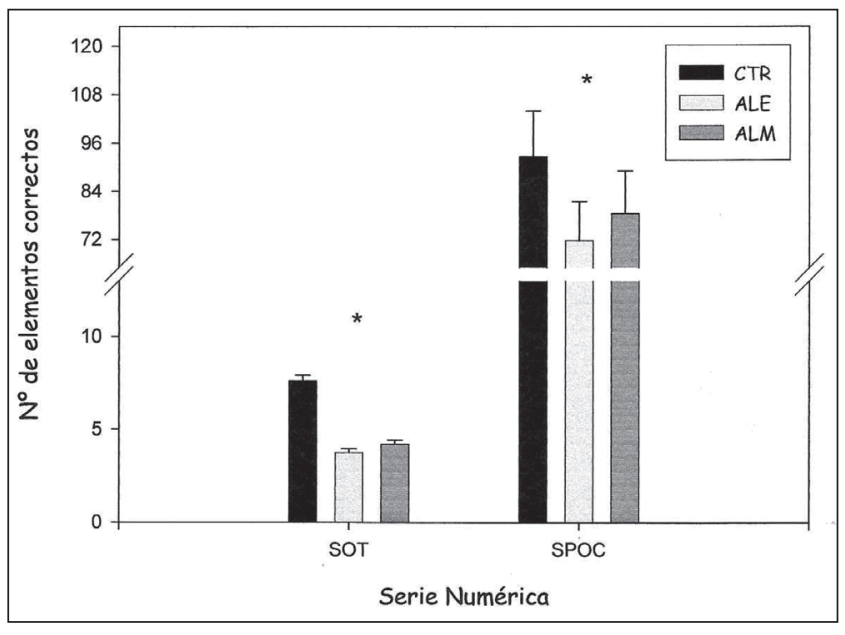

Figura 4. Cálculo mental. Resultados obtenidos en la serie numérica. Los sujetos con-trol son mejores tanto en el número de operaciones (SOT) como en el porcentaje de ellas realizadas correctamente (SPOC). (* Significatividad mínima $p<0$ '05).

\section{DISCUSIÓN}

Los resultados obtenidos en nuestro trabajo por los jóvenes que consumen alcohol de manera abusiva durante el fin de semana en relación con aquellos que no consumen ponen de manifiesto su peor rendimiento en tareas que requieren la participación de la corteza prefrontal y, de manera indirecta, los efectos negativos que el consumo excesivo de alcohol tiene sobre la misma. En estudios neuropatológicos realizados en cadáveres de alcohólicos y en estudios de Neuroimagen estructural en alcohólicos vivos, se ha puesto de manifiesto la mayor susceptibilidad de los circuitos frontales del cerebro a los efectos negativos del alcohol (Moselhy et al., 2001; Pfefferbaum et al., 1997; Sullivan, 2000). También con técnicas de neuroimagen funcional se ha observado un mayor efecto del alcohol sobre los lóbulos frontales. Por ejemplo, se ha encontrado una reducción del metabolismo de la glucosa en estas áreas en alcohólicos sin otras patologías neurológicas (Adams, Gilman, Koeppe, Kluin, Brunberg, Dede et al., 1993; Gilman, Adams, Koeppe, Berent, Kluin et al., 1990). Por otro lado, Jaatinen, Riikonen, Riihioja y Kajander (2003) demostraron que el consumo crónico intermitente de alcohol, el equivalente al consumo de fin de semana, ocasiona un descenso del metabolismo energético de las neuronas en la corteza prefrontal que contribuye al proceso de neurodegeneración y al consiguiente deterioro de las funciones cerebrales. Esto pone de manifiesto que el daño frontal en alcohólicos ha quedado demostrado con numerosos trabajos de investigación, pero, además también se ha observado que el deterioro neuropsicológico encontrado en alcohólicos crónicos es similar, en ciertos aspectos, al observado en pacien- 
tes con lesiones cerebrales localizadas en la corteza prefrontal (Beatty, Katzung, Nixon y Moreland, 1993; Ihara, Berrios y London, 2000; Joyce y Robbins, 1991; Tarter y Parsons, 1971) Ilevando incluso a formular la hipótesis del daño frontal como una de las explicaciones posibles al deterioro neuropsicológico observado en el alcoholismo. Esos resultados coinciden con los obtenidos en nuestro trabajo, con la diferencia de que, en este caso, el patrón de consumo ha sido distinto ya que no se trata de un alcoholismo crónico sino de un consumo crónico intermitente, es decir, alcoholismo de fin de semana.

La corteza prefrontal es la región neocortical más elaborada en los primates, animales que se caracterizan por poseer un repertorio conductual diverso y flexible, y, además, ocupa una posición privilegiada para coordinar un amplio rango de procesos neurales ya que está formada por un grupo de áreas interconectadas que envían y reciben proyecciones desde toda la corteza sensorial, motora y desde un buen número de estructuras subcorticales (Miller y Cohen, 2001). Diversos autores han demostrado que participa en el mantenimiento de la información necesaria para realizar una tarea o en la integración de información diversa y que puede manejar información procedente tanto del medio interno como del externo (Fuster, 1985 y 1989; Fuster, Bauer y Jervey, 1982 y 1985; Goldman-Rakic, 1987 y 1996; Goldman-Rakic, Selemon y Schwartz, 1984) y, por tanto, la corteza prefrontal se considera esencial para el correcto desarrollo de las Funciones Ejecutivas como han demostrado diferentes investigaciones en el ámbito de la Neuropsicología o la investigación clínica (Jodar-Vicente, 2004; Papazian et al., 2006). Su estudio en alcohólicos, con diferentes tareas que implican claramente a estas funciones, como el test de Stroop, el test de clasificación de cartas de Wisconsin o el BADS (Evaluación Conductual del Síndrome Disejecutivo), ha constatado la presencia de alteraciones que se pueden enmarcar en lo que se considera el Síndrome Disejecutivo (Field, Christiensen, Cole y Goudie, 2007; Ihara et al., 2000). Además, el hipometabolismo frontal encontrado en pacientes alcohólicos se correlaciona estrechamente con el rendimiento neuropsicológico de estos sujetos en funciones ejecutivas ya que se ha observado un menor rendimiento en tareas de categorización o flexibilidad cognitiva de sujetos alcohólicos desintoxicados con hipometabolismo frontal, incluso, sin que presenten atrofia cerebral (Adams et al., 1993; Nicolás, Catafau, Estruch, Lomena, Salamero-Herránz, Monforte et al., 1993)

Las alteraciones cerebrales ocasionadas por el consumo abusivo de alcohol pueden estar influidas por la presencia de determinados factores de riesgo o por el consumo de otras drogas de manera conjunta, sin embargo, un factor que determina en buena parte la presencia de estas alteraciones es la abstinencia alcohólica que prosigue al consumo, al menos, en sus fases iniciales (Neiman, 1998; Rathlev, Ulrich, Shieh, Callum, Bernstein y D'Onofrio, 2002; Rathlev et al., 2006). Una simple borrachera o un par de días de consumo elevado pueden ocasionar neurodegeneración en determinados circuitos cerebrales y provocar alteraciones cognitivas (Obernier, White, Swartzwelder y Crews, 2002), neurodegeneración que se manifiesta de forma temprana durante el consumo (Obernier, Bouldin y Crews, 2002) y que se puede ver agravada en las fases iniciales de la posterior abstinencia alcohólica manifestando sus efectos en el desempeño de los sujetos en diferentes tareas (Lukoyanov et al., 1996). El patrón de consumo en el alcoholismo de fin de semana implica una secuencia borrachera-abstinencia que se repite cada siete días, lo que implica que los jóvenes bebedores van a sufrir el daño neurocognitivo propio del consumo abusivo más el correspondiente al inicio de la abstinencia, y esto, con la regularidad que implica repetir el proceso prácticamente cada fin de semana.

El alcohol consumido de forma aguda provoca deterioro de las funciones cognitivas (Weissenborn y Duka, 2003) y este deterioro puede persistir en las fases iniciales de la abstinencia alcohólica (Zinn, Stein y Swartzwelder, 2004), lo cual nos ayuda a comprender la causa de que nuestros datos muestren un deterioro cognitivo en jóvenes cuya historia de consumo no es tan dilatada en el tiempo, equivalente al encontrado en consumidores crónicos con una historia de consumo de duración mucho mayor. Además, diversos estudios ponen de manifiesto que los adolescentes, cuyo cerebro esta aún en desarrollo y por tanto es más vulnerable, son más sensibles que los adultos a una gran parte de los efectos del alcohol, incluido el deterioro de los procesos cognitivos, especialmente, la memoria y el aprendizaje, y que esos efectos pueden continuar en la etapa adulta incluso habiendo dejado de consumir alcohol (Crews, Braun, Hoplight, Switzer y Knaapp; 2000; Crews et al., 2000; HillerSturmhofel y Swartzwelder, 2004; Tokunaga, Silvers y Matthews, 2006; Yttri, Buro y Hunt, 2004). Y es que la recuperación neurocognitiva después de un periodo de abstinencia no siempre ocurre en la medida deseada. El deterioro cognitivo en alcohólicos suele empeorar en la primera semana de abstinencia, aunque posteriormente, tiende a remitir experimentandose una mejoría mientras se mantenga la abstinencia. Esta recuperación, referida a un buen número de procesos cognitivos, puede proseguir en su mejoría hasta equipararse en rendimiento al de controles no alcohólicos (Redd, Grant y Rourke, 1992; Rourke y Grant, 1999). No obstante, también se ha observado la persistencia de deterioro en algunos procesos cognitivos después de una abstinencia prolongada. Parece que 
se produce una rápida recuperación de algunas funciones neuropsicológicas mientras que otras son más resistentes a la recuperación (Mann, Günter, Stetter y Ackermann, 1999), por ejemplo, la memoria y las funciones ejecutivas, cuya recuperación es más lenta e incompleta (Munro, Saxton y Butters, 2000).

Los datos de nuestra investigación apoyan la idea de que el consumo elevado de alcohol por parte de los jóvenes en fin de semana ocasiona daños en la corteza prefrontal del cerebro que se traducen en una alteración significativa de las funciones ejecutivas, capacidades que se requieren para la organización de la conducta y el diseño de estrategias encaminadas a lograr nuestros objetivos. El consumo abusivo de manera intermitente puede acelerar el proceso de deterioro, ya que alterna secuencias consumoabstinencia que incrementan los efectos neurotóxicos del etanol sobre el cerebro. La abstinencia mantenida puede acarrear una mejora de la cognición pero en el caso de las funciones ejecutivas corremos el riesgo de que esa recuperación, si no se toman medidas urgentes, no llega a producirse de manera completa incluso después de un largo periodo de abstinencia total. En este sentido, los jóvenes que han convertido el consumo de alcohol en un fin más que en un medio deben conocer la manera en que sus vidas pueden quedar afectadas para el futuro.

\section{AGRADECIMIENTOS}

El presente trabajo ha sido financiado mediante una ayuda concedida por el Ministerio del Interior a través de la Delegación del Gobierno para el Plan Nacional sobre Drogas (Ref. PR153/03-12277) y por otra ayuda concedida por Banco Santander-Universidad Complutense (Ref. PR27/05-14004).

\section{REFERENCIAS}

Adams, K.M., Gilman, S., Koeppe, R.A., Kluin, K.J., Brunberg, J.A., Dede, D., et al. (1993). Neuropsychological deficits are correlated with frontal hypometabolism in positron emission tomography studies of older alcoholic patients. Alcohol: Clinical and Experimental Research, 17, 205-210.

Beatty, W.W., Katzung, V.M., Nixon, S.J. y Moreland, V.J. (1993). Problem-solving deficits in alcoholics: evidence from the California Card Sorting Test. Journal of Studies on Alcohol, 54, 687-692.

Bechara, A., Damasio, H. y Damasio, A.R. (2000). Emotion, decision-making and the orbitofrontal cortex. Cerebral Cortex, 10, 295-307.
Beracochea, D., Durkin, T.P. y Jaffard, R. (1986). On the involvement of the central cholinergic system in memory deficits induced by long-term ethanol consumption in mice. Pharmacology, Biochemistry and Behavior, 24, 519-524.

Cadaveira, F. y Corral-Varela, M. (2005). Alcohol y cerebro: efectos de los nuevos patrones de consumo. En: I. Morgado (Coord.), Psicobiología: de los Genes a la Cognición y el Comportamiento (pp. 145-153). BarceIona: Ariel.

Calafat, A., Juan, M., Becoña, E., Castillo, A., Fernández, C., Franco, M., et al. (2005). El consumo de alcohol en la lógica del botellón. Adicciones, 17, 103-202.

Carpenter-Hyland, E. y Chandler, L. (2007). Adaptative plasticity of NMDA receptors and dendritic spines: Implications for enhanced vulnerability of the adolescent brain to alcohol addiction. Pharmacology Biochemistry and Behavior, 86, 200-208.

Charness, M.E. (1993). Brain lesions in alcoholics. Alcohol: Clinical and Experimental Research, 17, 2-11.

Corral-Varela, M. y Cadaveira, F. (2002). Aspectos neuropsicológicos de la dependencia del alcohol: naturaleza y reversibilidad del daño cerebral. Revista de Neurología, 35, 682-687.

Crews, F., He, J. y Hodge, C. (2007). Adolescent cortical development: A critical period of vulnerability for addiction. Pharmacology, Biochemistry and Behavior, 86, 189-199.

Crews, F.T., Braun, C.J., Hoplight, B., Switzer, R.C. y Knaapp, D.J. (2000). Binge ethanol consumption causes differential brain damage in young adolescent rats compared with adult rats. Alcohol: Clinical and Experimental Research, 24, 1712-1723.

Cummings, J.L. (1993). Frontal subcortical circuits and human behavior. Archives of Neurology, 50, 873-80.

Damasio, A. (1996). El error de Descartes. La emoción, la razón y el cerebro humano. Barcelona: Crítica.

Field, M., Christiensen, P., Cole, J. y Goudie, A. (2007). Delay discounting and the alcohol Stroop in heavy drinking adolescent. Addiction, 102, 579-586.

Fuster, J.M. (1985). The prefrontal cortex, mediator of crosstemporal contingencies. Human Neurobiology, 4, 169-179.

Fuster, J.M. (1989). The Prefrontal Cortex. New York: Raven

Fuster, J.M., Bauer, R.H. y Jervey, J.P. (1982). Cellular discharge in the dorsolateral prefrontal cortex of the monkey in cognitive tasks. Experimental Neurology, 77, 679-694.

Fuster, J.M., Bauer, R.H. y Jervey, J.P. (1985). Functional interactions between inferotemporal and prefrontal cortex in a cognitive task. Brain Research, 330, 299-307.

Garrido, M.J. y Fernández-Guinea, S. (2004). Déficit neuropsicológicos en alcohólicos: implicaciones para la seguridad vial. Revista de Neurología, 38, 277-283.

Gilman, S., Adams, K., Koeppe, R.A., Berent, S., Kluin, K.J., Modell, J.G., et al. (1990). Cerebellar and frontal hypometabolism in alcoholic cerebellar degeneration 
studied with positron emission tomography. Annals of Neurology, 28, 775-785.

Goldman-Rakic, P.S. (1987). Circuitry of primate prefrontal cortex and regulation of behavior by representational memory. En: F. Plum (ed.), Handbook of Physiology: The Nervous System (pp. 373-417). Bethesda, MD: APS Books.

Goldman-Rakic, P.S. (1996). The prefrontal landscape: implications of functional architecture for understanding human mentation and the central executive. Philosophical Transactions of the Royal Society of London. Series B, Biological Sciences, 351, 1445-1453.

Goldman-Rakic, P.S., Selemon, L.D. y Schwartz, M.L. (1984). Dual pathways connecting the dorsolateral prefrontal cortex with the hippocampal formation and parahippocampal cortex in the rhesus monkey. Neuroscience, 12, 719-743.

Hiller-Sturmhofel, S. y Swartzwelder, H.S. (2004). Alcohol's effects on the adolescent brain - What can be learned from animal models. Alcohol Research and Health, 28, 213-221.

Ihara, H., Berrios, G.E. y London, M. (2000). Group and case study of the dysexecutive syndrome in alcoholics without amnesia. Journal of Neurology, Neurosurgery and Psychiatry, 68, 731-737.

Jaatinen, P., Riikonen, J., Riihioja, P. y Kajander, O. (2003). Interaction of aging and intermittent ethanol exposure on brain cytochrome c oxidase activity levels. Alcohol, 29, 91-100.

Jodar-Vicente, M. (2004). Funciones cognitivas del lóbulo frontal. Revista de Neurología, 39, 178-182.

Joyce, E.M. y Robbins, T.W. (1991). Frontal lobe function in Korsakoff and non-Korsakoff alcoholics: planning and spatial working memory. Neuropsychologia, 8, 709-723.

Landa, N., Fernández-Montalvo, J. y Tirapu, J. (2004). Alteraciones neuropsicológicas en el alcoholismo: una revisión sobre la afectación de la memoria y las funciones ejecutivas. Adicciones, 16, 41-52.

Lukoyanov, N.V., Madeira, M.D. y Paula-Barbosa, M.M. (1996). Behavioral and neuroanatomical consequences of chronic ethanol intake and withdrawal. Physiology and Behavior, 66, 337-346.

Mann, K., Günter, A., Stetter, F. y Ackermann, K. (1999). Rapid recovery from cognitive defects in abstinent alcoholics: a controlled test-retest study. Alcohol and Alcoholism, 34, 567-74.

Miller, E.H. y Cohen, J.D. (2001). An integrative theory of prefrontal cortex function. Annual Review of Neuroscience, 24, 167-202.

Moselhy, H.F., Georgiou, G. y Kahn, A. (2001). Frontal lobe changes in alcoholism: A review of the literature. Alcohol and Alcoholism, 36, 357-368.

Munro, C.A., Saxton, J. y Butters, M.A. (2000). The neuropsychological consequences of abstinence among older alcoholics a cross-sectional study. Alcohol: Clinical and Experimental Research, 24, 1510-1516.
Neiman, J. (1998). Alcohol as a risk factor for brain damage. Neurologic aspects. Alcohol: Clinical and Experimental Research, 22(Suppl), 346-351.

Nicolás, J.M., Catafau, A.M., Estruch, R., Lomena, F.J., Salamero-Herránz, R., Monforte, R., et al. (1993). Regional cerebral blood flow-SPECT in chronic alcoholism: relation to neuropsychological testing. Journal of Nuclear Medicine, 34, 1452-1459.

Obernier, J.A., Bouldin, T.W. y Crews, F.T. (2002). Binge ethanol exposure in adult rats causes necrotic cell death. Alcohol: Clinical and Experimental Research, 26, 547-557.

Obernier, J.A., White, A.M., Swartzwelder, H.S. y Crews, F.T. (2002). Cognitive deficits and CNS damage after a 4-day binge ethanol exposure in rats. Pharmacology, Biochemistry and Behavior, 72, 521-532.

Papazian, O., Alfonso, I. y Luzondo, R.J. (2006). Trastornos de las funciones ejecutivas. Revista de Neurología, 42 (Sup/ 3), 45-50.

Pascual, F. (2002). Percepción del alcohol entre los jóvenes. Adicciones, 14 (Sup/ 1), 124-131.

Pascual, M., Blanco, A.M., Cauli, O., Minarro, J. y Guerri, C. (2007). Intermittent ethanol exposure induces inflammatory brain damage and causes long-term behavioural alterations in adolescent rats. European Journal of Neuroscience, 25, 541-550.

Pfefferbaum, M.A., Sullivan, E.V., Mathalon, D.H. y Lim, K.O. (1997). Frontal lobe volume loss observed with magnetic resonance imaging in older chronic alcoholics. Alcohol: Clinical and Experimental Research, 21, 521-529.

Rathlev, N.K., Ulrich, A.S., Delanty, N. y D'Onofrio, G. (2006). Alcoho-related seizures. The Journal of Emergency Medicine, 31, 157-163.

Rathlev, N.K., Ulrich, A., Shieh, T.C., Callum, M.G., Bernstein, E. y D'Onofrio, G. (2002). Etiology and weekly occurrence of alcohol-related seizures. Academic Emergency Medicine, 9, 824-828.

Redd, R.J., Grant, I. y Rourke, S.B. (1992). Long-term abstinent alcoholics have normal memory. Alcohol: Clinical and Experimental Research, 16, 677-83.

Roehrich, L. y Goldman M.S. (1993). Experience-dependent neuropsychological recovery and the treatment of alcoholism. Journal of Consulting and Clinical Psychology, 61, 812-821.

Rosenbloom, M., Sullivan, E.V. y Pfefferbaum, A. (2003). Using Magnetic Resonance Imaging and Difusión Tensor Imaging to assess brain damage in alcoholics. Alcohol Research and Health, 27, 146-152.

Rourke, S.B. y Grant, I. (1999). The interactive effects of age and length of abstinence on the recovery of neuropsychological functioning in chronic male alcoholics: a 2-year follow-up study. Journal of the International Neuropsychological Society, 5, 234-46.

Sánchez, L. (2002) Consumo alcohólico en la población española. Adicciones, 14 (Sup/ 1), 81-100. 
Stuss, D.T. y Alexander, M.P. (2000). Executive functions and the frontal lobes: a conceptual view. Psychological Research, 63, 289-98.

Sullivan, E.V. (2000). Human brain vulnerability to alcoholism: Evidence from neuroimaging studies. En: V. Noronha, M. Eckardt y K. Warren (eds.), Review of NIAAA's Neuroscience and Behavioral Research Portfolio. NIAAA Research Monograph $n^{\circ} 34$ (pp 473-508). Bethesda, MD: National Institute of Health.

Tarter, R.E. y Parsons, O.A. (1971). Conceptual shifting in chronic alcoholics. Journal of Abnormal Psychology, 77, 71-75.

Tirapu-Ustárroz, J., Muñoz-Céspedes, J.M., PelegrínValero, C. y Albéniz-Ferreras, A. (2005). Propuesta de un protocolo para la evaluación de las funciones ejecutivas. Revista de Neurología, 41, 177-186.

Tokunaga, S., Silvers, J.M. y Matthews, D.B. (2006). Chronic intermittent ethanol exposure during adolescence blocks ethanol-induced inhibition of spontaneously active hippocampal pyramidal neurons. Alcohol: Clinical and Experimental Research, 30, 1-6.

Verdejo, A., Orozco, C., Meersmans, M., Aguilar, F. y PérezGarcía, M. (2004). Impacto de la gravedad del consumo de drogas sobre distintos componentes de la función ejecutiva. Revista de Neurología, 38, 1109-1116.

Victor, M. (1994). Alcoholic dementia. The Canadian Journal of Neurological Sciences, 21, 88-99.

Walker, D.W., Hunter, B.E. y Abraham, W.C. (1981). Neuroanatomical and functional deficits subsequent to chronic ethanol administration in animals. Alcohol: Clinical and Experimental Research, 5, 267-282.

Weissenborn, R. y Duka, T. (2003). Acute alcohol effects on cognitive function in social drinkers: their relationship to drinking habits. Psychopharmacology, 165, 306-312.

Yttri, E.A., Buro, J.A. y Hunt, P.S. (2004). Intermittent ethanol exposure in adolescent rat: Dose-dependent impairments in trace conditioning. Alcohol: Clinical and Experimental Research, 28, 1433-1436.

Zeigler, D.W., Wang, C.C., Yoast, R.A., Dickinson, B.D., McCaffree, M.A., Robinowitz, C.B., et al. (2005). The neurocognitive effects of alcohol on adolescents and college students. Preventive Medicine, 40, 23-32.

Zinn, S., Stein, R. y Swartzwelder, H.S. (2004). Executive functioning early in abstinence from alcohol. Alcohol: Clinical and Experimental Research, 28, 1338-1346. 
